\title{
POSSIBLE ISOLATION NUMBER OF A MATRIX OVER NONNEGATIVE INTEGERS
}

\author{
LeRoy B. Beasley, Logan, Young Bae Jun, Jinju, Seok-Zun Song, Jeju
}

Received February 17, 2017. Published online May 8, 2018.

\begin{abstract}
Let $\mathbb{Z}_{+}$be the semiring of all nonnegative integers and $A$ an $m \times n$ matrix over $\mathbb{Z}_{+}$. The rank of $A$ is the smallest $k$ such that $A$ can be factored as an $m \times k$ matrix times a $k \times n$ matrix. The isolation number of $A$ is the maximum number of nonzero entries in $A$ such that no two are in any row or any column, and no two are in a $2 \times 2$ submatrix of all nonzero entries. We have that the isolation number of $A$ is a lower bound of the rank of $A$. For $A$ with isolation number $k$, we investigate the possible values of the rank of $A$ and the Boolean rank of the support of $A$. So we obtain that the isolation number and the Boolean rank of the support of a given matrix are the same if and only if the isolation number is 1 or 2 only. We also determine a special type of $m \times n$ matrices whose isolation number is $m$. That is, those matrices are permutationally equivalent to a matrix $A$ whose support contains a submatrix of a sum of the identity matrix and a tournament matrix.
\end{abstract}

Keywords: rank; Boolean rank; isolated entry; isolation number

MSC 2010: 15A23, 15A03, 15B34

\section{INTRODUCTION AND PRELIMINARIES}

A semiring is a set $\mathbb{S}$ with two binary operations, "+" and "." such that $(\mathbb{S},+)$ is an abelian monoid with identity $0,(\mathbb{S}, \cdot)$ is a monoid with identity 1 , such that "." distributes over "+" from both sides and such that $0 \cdot s=s \cdot 0=0$ for all $s \in \mathbb{S}$. We use juxtaposition for "." for convenience. Let $\mathbb{B}$ be the binary Boolean semiring, that is, $\mathbb{B}=\{0,1\}$ with addition and multiplication defined as in the real numbers except that $1+1=1$. The set, $\mathbb{Z}_{+}$, of nonnegative integers and $\mathbb{B}$, the binary Boolean semiring, are examples of combinatorially interesting semirings.

Let $\mathcal{M}_{m, n}\left(\mathbb{Z}_{+}\right)$denote the set of all $m \times n$ matrices with entries in $\mathbb{Z}_{+}$with matrix

This research was supported by Basic Science Research Program through the National Research Foundation of Korea (NRF) funded by the Ministry of Education (2016R1D1A1B02006812). 
addition and multiplication following the usual rules. Let $\mathcal{M}_{n}\left(\mathbb{Z}_{+}\right)=\mathcal{M}_{m, n}\left(\mathbb{Z}_{+}\right)$if $m=n$, let $I_{m}$ denote the $m \times m$ identity matrix, $O_{m, n}$ denote the $m \times n$ zero matrix, $J_{m, n}$ denote the $m \times n$ matrix of all ones. The subscripts are usually omitted if the order is obvious, and we write $I, O, J$.

The matrix $A \in \mathcal{M}_{m, n}\left(\mathbb{Z}_{+}\right)$is said to be of rank $k$, see [2], if there exist matrices $B \in \mathcal{M}_{m, k}\left(\mathbb{Z}_{+}\right)$and $C \in \mathcal{M}_{k, n}\left(\mathbb{Z}_{+}\right)$such that $A=B C$ and $k$ is the smallest positive integer that such a factorization exists. We denote $r_{\mathbb{Z}_{+}}(A)=k$.

Now let $\mathcal{M}_{m, n}(\mathbb{B})$ denote the set of all $m \times n$ Boolean matrices with entries in $\mathbb{B}$. The matrix $A \in \mathcal{M}_{m, n}(\mathbb{B})$ is said to be of Boolean rank $k$ (see [5]) if there exist matrices $B \in \mathcal{M}_{m, k}(\mathbb{B})$ and $C \in \mathcal{M}_{k, n}(\mathbb{B})$ such that $A=B C$ and $k$ is the smallest positive integer that such a factorization exists. We denote $b(A)=k$. Then $r_{\mathbb{Z}_{+}}(O)=$ $b(O)=0$, and $O$ is the only matrix of (Boolean) rank 0 .

The Boolean rank has many applications in combinatorics, especially the graph theory, for example, if $A \in \mathcal{M}_{m, n}(\mathbb{B})$ is the adjacency matrix of a bipartite graph $G$ with bipartition $(X, Y)$, the Boolean rank of $A$ is the minimum number of bicliques that cover the edges of $G$, called the biclique covering number (see [6]). If $A \in$ $\mathcal{M}_{m, n}\left(\mathbb{Z}_{+}\right)$, define a Boolean $m \times n$ matrix $\bar{A}=\left(\bar{a}_{i, j}\right)$ such that $\bar{a}_{i, j}=1$ if and only if $a_{i, j} \neq 0$. We call $\bar{A}$ the support of $A$. Then "-" maps $\mathcal{M}_{m, n}\left(\mathbb{Z}_{+}\right)$onto $\mathcal{M}_{m, n}(\mathbb{B})$, and preserves matrix addition, multiplication, and multiplication by scalars. That is, "-" is a homomorphism.

We say that a matrix $A$ dominates a matrix $B$ if $a_{i, j}=0$ implies $b_{i, j}=0$.

Given a matrix $X$, we let $\mathbf{x}^{(j)}$ denote the $j$ th column of $X$ and $\mathbf{x}_{(i)}$ denote the $i$ th row. Now if $r_{\mathbb{Z}_{+}}(A)=k$ and $A=B C$ is a factorization of $A \in \mathcal{M}_{m, n}\left(\mathbb{Z}_{+}\right)$, then $A=$ $\mathbf{b}^{(1)} \mathbf{c}_{(1)}+\mathbf{b}^{(2)} \mathbf{c}_{(2)}+\ldots+\mathbf{b}^{(k)} \mathbf{c}_{(k)}$. Since each of the terms $\mathbf{b}^{(i)} \mathbf{c}_{(i)}$ is a rank one matrix, the rank of $A$ is also the minimum number of rank one matrices whose sum is $A$.

Given a matrix $A \in \mathcal{M}_{m, n}\left(\mathbb{Z}_{+}\right)$, a set of isolated entries (see [6]) is a set of locations, usually written as $I=\left\{a_{i, j}\right\}$ such that $a_{i, j} \neq 0$, no two entries in $I$ are in the same row, no two entries in $I$ are in the same column, and, if $a_{i, j}, a_{k, l} \in I$, then $a_{i, l}=0$ or $a_{k, j}=0$. That is, isolated entries are independent entries and no two isolated entries $a_{i, j}$ and $a_{k, l}$ lie in a submatrix of $A$ of the form $\left[\begin{array}{cc}a_{i, j} & a_{i, l} \\ a_{k, j} & a_{k, l}\end{array}\right]$ with all entries nonzero. The isolation number of $A, \iota(A)$, is the maximum size of a set of isolated entries. Note that $\iota(A)=0$ if and only if $A=O$.

Example 1.1. Let

$$
A=\left[\begin{array}{lllll}
1 & 1 & 2 & 0 & 0 \\
2 & 1 & 0 & 1 & 0 \\
1 & 0 & 0 & 0 & 2 \\
0 & 2 & 0 & 1 & 1 \\
0 & 0 & 1 & 2 & 1
\end{array}\right]
$$


and $S=\{(1,3),(2,1),(3,5),(4,2),(5,4)\}$. The entries (2's) of $A$ with positions in $S$ are isolated entries and hence $\iota(A)=5$. But the entries of $A$ in the position in $T=((1,1),(2,2),(3,5),(4,4),(5,3))$ are not isolated.

Suppose that $A \in \mathcal{M}_{m, n}\left(\mathbb{Z}_{+}\right)$and $r_{\mathbb{Z}_{+}}(A)=k$. Then there are $k$ rank one matrices $A_{i}$ such that

$$
A=A_{1}+A_{2}+\ldots+A_{k} .
$$

Because each rank one matrix can be permuted to a matrix of the form $\left[\begin{array}{ll}B & O \\ O & O\end{array}\right]$ with $\bar{B}=J$, it is easily seen that the matrix consisting of two isolated entries of $A$ cannot be dominated by any $A_{i}$ among the rank one summand of $A$ in (1.1). Thus

$$
\iota(A) \leqslant r_{\mathbb{Z}_{+}}(A) .
$$

For $A=A_{1}+A_{2}+\ldots+A_{k}$, let $\mathcal{R}_{i}$ denote the indices of the nonzero rows of $A_{i}$ and $\mathcal{C}_{j}$ denote the indices of the nonzero columns of $A_{j}, i, j=1, \ldots, k$. Let $r_{i}=\left|\mathcal{R}_{i}\right|$, the number of nonzero rows of $A_{i}$ and $c_{j}=\left|\mathcal{C}_{j}\right|$, the number of nonzero columns of $A_{j}$.

Many functions, sets and relations concerning matrices do not depend upon the magnitude or nature of the individual entries of a matrix, but rather only on whether the entry is zero or nonzero. These combinatorially significant matrices have become increasingly important in recent years. Of primary interest is the Boolean rank. Finding the Boolean rank of a (0,1)-matrix is an NP-Complete problem (see [8]), and consequently finding bounds on the Boolean rank of a matrix is of interest to those researchers that would use Boolean rank in their work. If the $(0,1)$-matrix is the reduced adjacency matrix of a bipartite graph, the isolation number of the matrix is the maximum size of a non-competitive matching in the bipartite graph. This is related to the study of such combinatorial problems as the patient hospital problem, the stable marriage problem, etc. An additional reason for studying the isolation number is that it is a lower bound on the rank of a matrix over $\mathbb{Z}_{+}$. While finding the isolation number as well as finding the rank of a matrix is an NP-Complete problem (see [7]), for some matrices finding the isolation number can be easier than finding the rank especially if the matrix is sparse. As the following example shows, the isolation number can be helpful to determine the rank of a given matrix. 
Example 1.2. Let

$$
B=\left[\begin{array}{lllllllll}
1 & 1 & 1 & 3 & 0 & 1 & 1 & 1 & 1 \\
1 & 1 & 1 & 1 & 3 & 1 & 1 & 1 & 1 \\
1 & 1 & 1 & 0 & 0 & 3 & 1 & 1 & 1 \\
1 & 1 & 1 & 0 & 0 & 0 & 0 & 0 & 0 \\
1 & 1 & 1 & 0 & 0 & 0 & 0 & 0 & 0 \\
1 & 1 & 1 & 0 & 0 & 0 & 0 & 0 & 0 \\
3 & 1 & 0 & 0 & 0 & 0 & 0 & 0 & 0 \\
0 & 3 & 1 & 0 & 0 & 0 & 0 & 0 & 0 \\
1 & 0 & 3 & 0 & 0 & 0 & 0 & 0 & 0
\end{array}\right]
$$

Then we can easily see $r_{\mathbb{Z}_{+}}(B) \leqslant 6$ from the first 3 rows and columns, however to find that it is not 5 , requires much calculation if the isolation number is not considered. However, the isolation number is easily seen to be 6 , both computationally and visually, the 3's in the matrix represent a set of isolated entries. Thus we have $r_{\mathbb{Z}_{+}}(B)=6$ by $(1.2)$.

Note that if any of the 1's in $B$ are replaced by zeros, the resulting matrix still has rank 6 as well as isolation number 6 .

Terms not specifically defined here can be found by Brualdi and Ryser, see [4], for matrix terms, or Bondy and Murty, see [3], for graph theoretic terms.

Beasley and Pullman in [2] introduced rank of a matrix in $\mathcal{M}_{m, n}\left(\mathbb{Z}_{+}\right)$and compared it with Boolean rank of its support. Gregory et al. in [6] introduced the set of isolated entries and compared Boolean rank with biclique covering number. Beasley in [1] introduced isolation number of Boolean matrix and compared it with Boolean rank.

In this paper, we investigate the question: Given a fixed $k$, if the isolation number of $A$ is $k$ what are some of the possible values of the rank of $A$ and the Boolean rank of the support of $A$ ? So we obtain that the isolation number and the Boolean rank of the support of a given matrix are the same if and only if the isolation number is 1 or 2 only. We also obtain a special type of $m \times n$ matrices whose isolation number is $m$. That is, those matrices are permutationally equivalent to a matrix $A$ whose support contains a submatrix of a sum of the identity matrix of order $m$ and a tournament matrix.

In the extensive researches on isolation numbers of matrices over $\mathbb{Z}_{+}$we shall apply this research to characterize the linear operators that preserve the isolation numbers of matrices over $\mathbb{Z}_{+}$. 


\section{Possible bounds for isolation numbers of a matrix in $\mathcal{M}_{m, n}\left(\mathbb{Z}_{+}\right)$}

In this section, we compare the isolation number with rank of a matrix in $\mathcal{M}_{m, n}\left(\mathbb{Z}_{+}\right)$, and we compare the isolation number with Boolean rank of the support of a matrix in $\mathcal{M}_{m, n}\left(\mathbb{Z}_{+}\right)$.

Lemma 2.1. For $A, B \in \mathcal{M}_{m, n}\left(\mathbb{Z}_{+}\right), r_{\mathbb{Z}_{+}}(A+B) \leqslant r_{\mathbb{Z}_{+}}(A)+r_{\mathbb{Z}_{+}}(B)$. And for $A, B \in \mathcal{M}_{m, n}(\mathbb{B}), b(A+B) \leqslant b(A)+b(B)$.

Lemma 2.2. For $A, B \in \mathcal{M}_{m, n}\left(\mathbb{Z}_{+}\right), \overline{A+B}=\bar{A}+\bar{B}$ in $\mathcal{M}_{m, n}(\mathbb{B})$.

Proof. It follows from the facts that all the entries of $A, B \in \mathcal{M}_{m, n}\left(\mathbb{Z}_{+}\right)$are nonnegative and $1+1=1$ in $\mathbb{B}$.

Lemma $2.3([6])$. For $A \in \mathcal{M}_{m, n}\left(\mathbb{Z}_{+}\right), b(\bar{A}) \leqslant r_{\mathbb{Z}_{+}}(A)$.

Proof. If $r_{\mathbb{Z}_{+}}(A)=k$, then $A$ has a rank-one factorization such that $A=$ $\mathbf{b}^{(1)} \mathbf{c}_{(1)}+\mathbf{b}^{(2)} \mathbf{c}_{(2)}+\ldots+\mathbf{b}^{(k)} \mathbf{c}_{(k)}$ with $B=\left[\mathbf{b}^{(1)} \mathbf{b}^{(2)} \ldots \mathbf{b}^{(k)}\right] \in \mathbf{M}_{m, k}\left(\mathbb{Z}_{+}\right)$and $C=\left[\mathbf{c}_{(1)} \mathbf{c}_{(2)} \ldots \mathbf{c}_{(k)}\right]^{t} \in \mathbf{M}_{k, n}\left(\mathbb{Z}_{+}\right)$. Therefore

$$
\begin{aligned}
b(\bar{A}) & =b\left(\overline{\mathbf{b}^{(1)} \mathbf{c}_{(1)}+\mathbf{b}^{(2)} \mathbf{c}_{(2)}+\ldots+\mathbf{b}^{(k)} \mathbf{c}_{(k)}}\right) \\
& =b\left(\overline{\mathbf{b}^{(1)} \mathbf{c}_{(1)}}+\overline{\mathbf{b}^{(2)} \mathbf{c}_{(2)}}+\ldots+\overline{\mathbf{b}^{(k)} \mathbf{c}_{(k)}}\right) \leqslant k,
\end{aligned}
$$

from Lemma 2.2 .

Hence $b(\bar{A}) \leqslant r_{\mathbb{Z}_{+}}(A)$.

We may have $b(\bar{A})<r_{\mathbb{Z}_{+}}(A)$ for some $A \in \mathcal{M}_{m, n}\left(\mathbb{Z}_{+}\right)$.

Example 2.4. Consider

$$
A=\left[\begin{array}{ll}
1 & 2 \\
3 & 4
\end{array}\right] \quad \text { and } \quad B=\left[\begin{array}{ll}
1 & 2 \\
3 & 6
\end{array}\right]
$$

in $\mathcal{M}_{2,2}\left(\mathbb{Z}_{+}\right)$. Then $r_{\mathbb{Z}_{+}}(A)=2$ but $b(\bar{A})=b\left(\left[\begin{array}{ll}1 & 1 \\ 1 & 1\end{array}\right]\right)=1$. Hence $b(\bar{A})<r_{\mathbb{Z}_{+}}(A)$. But $r_{\mathbb{Z}_{+}}(B)=b(\bar{B})=1$.

Lemma 2.5. For $A=\left[a_{i, j}\right] \in \mathcal{M}_{m, n}\left(\mathbb{Z}_{+}\right), \iota(A)=\iota(\bar{A})$.

P r o of. If $a_{i, j}$ and $a_{k, l}$ are any isolated entries in $A$, then $i \neq k$ and $j \neq l$, and then $a_{i, l}=0$ or $a_{k, j}=0$. Hence $\bar{a}_{i, j}$ and $\bar{a}_{k, l}$ are isolated entries in $\bar{A}$, so we have $\iota(A) \leqslant \iota(\bar{A})$.

Conversely, if $\bar{a}_{i, j}$ and $\bar{a}_{k, l}$ are any isolated entries in $\bar{A}$, then $a_{i, j} \neq 0$ and $a_{k, l} \neq 0$ and then $a_{i, l}=\bar{a}_{i, l}=0$ or $a_{k, j}=\bar{a}_{k, j}=0$. Hence $a_{i, j}$ and $a_{k, l}$ are isolated entries in $A$, so we have $\iota(\bar{A}) \leqslant \iota(A)$. 
Theorem 2.6. If $A \in \mathcal{M}_{m, n}\left(\mathbb{Z}_{+}\right)$, then $\iota(A)=1$ if and only if $b(\bar{A})=1$.

Pr o of. Let $A \in \mathcal{M}_{m, n}\left(\mathbb{Z}_{+}\right)$. If $b(\bar{A})=1$ then $A \neq O$ so that $\iota(A) \neq 0$ and since $\iota(A)=\iota(\bar{A}) \leqslant b(\bar{A})$ by $(1.2)$, we have $\iota(A)=1$.

Conversely, suppose that $\iota(A)=1$ and that $b(\bar{A}) \geqslant 2$. Then, for some permutation matrices $P$ and $Q$ of the appropriate orders, $P \bar{A} Q=\left[\begin{array}{cc}J_{r, s} & O \\ O & O\end{array}\right]+\bar{A}_{2}$ for some $r, s$ with either $r<m$ or $s<n$. Partition $\bar{A}_{2}$ as $\bar{A}_{2}=\left[\begin{array}{ll}\bar{A}_{2,1} & \bar{A}_{2,2} \\ \bar{A}_{2,3} & \bar{A}_{2,4}\end{array}\right]$, where $\bar{A}_{2,1}$ is $r \times s$. Since $b(P \bar{A} Q)=b(\bar{A}) \geqslant 2$, we have $\bar{A} \neq J$, and hence, one of $\bar{A}_{2,2}, \bar{A}_{2,3}, \bar{A}_{2,4}$ has a zero entry. Further, one of $\bar{A}_{2,2}, \bar{A}_{2,3}, \bar{A}_{2,4}$ has an entry of 1 since $P \bar{A} Q \neq\left[\begin{array}{cc}J_{r, s} & O \\ O & O\end{array}\right]$. Thus, in $P \bar{A} Q$, there is some zero entry, say $\bar{a}_{i, j}=0$, which lies in a nonzero column $j$ and a nonzero row $i$. Then, any of the ones in that column $j$ together with a one in the nonzero row $i$ form a set of two isolated entries, a contradiction, thus $b(\bar{A})=1$.

It follows that the subset of $\mathcal{M}_{m, n}\left(\mathbb{Z}_{+}\right)$of matrices with isolation number one is the same as the set of matrices whose support has Boolean rank one.

Lemma 2.7. Let $A \in \mathcal{M}_{m, n}\left(\mathbb{Z}_{+}\right)$. Then if $r_{\mathbb{Z}_{+}}(A) \geqslant b(\bar{A})=2$ then $\iota(A)=2$, and if $\iota(A)=2$ then $b(\bar{A}) \neq 3$.

Proof. If $b(\bar{A})=2$, then $\iota(A)>1$ by Theorem 2.6. Since $\iota(A)=\iota(\bar{A}) \leqslant b(\bar{A})$ from Lemma 2.5 and $(1.2)$, we have that $\iota(A)=\iota(\bar{A})=2$.

Now, suppose that $\iota(A)=2$ and that $b(\bar{A})=3$. Let $\bar{A}=\bar{A}_{1}+\bar{A}_{2}+\bar{A}_{3}$ where $b\left(\bar{A}_{i}\right)=1$.

Permute the rows of $\bar{A}$ so that $\mathcal{R}_{1}=\left\{1,2, \ldots, r_{1}\right\}$ and permute the columns of $\bar{A}$ so that $\mathcal{C}_{2}=\left\{1,2, \ldots, c_{2}\right\}$ and $\mathcal{C}_{3}=\left\{k+1, k+2, \ldots, k+c_{3}\right\}$ where $k \leqslant c_{2}$.

Note that $\mathcal{R}_{i} \neq \mathcal{R}_{j}$ and $\mathcal{C}_{i} \neq \mathcal{C}_{j}$ unless $i=j$, otherwise $\bar{A}_{i}+\bar{A}_{j}$ would be rank 1 .

Suppose that $\mathcal{R}_{1} \subset \mathcal{R}_{2}$. Permute the remaining rows so that $\mathcal{R}_{2}=\left\{1,2, \ldots, r_{2}\right\}$, and $\mathcal{R}_{3}=\left\{a+1, a+2, \ldots, a+b+c, r_{2}+1, r_{2}+2, \ldots, r_{2}+e\right\}$ where $a+b \leqslant r_{1}$, $r_{1} \leqslant a+b+c \leqslant a+b+c+d \leqslant r_{2}$ and $r_{2} \leqslant a+b+c+d+e$.

Thus, we have that

$$
\bar{A}=\left[\begin{array}{cccccc}
J_{a, k} & J_{a, g} & J_{a, h} & O_{a, u} & J_{a, v} & O_{a, w} \\
J_{b, k} & J_{b, g} & J_{b, h} & J_{b, u} & J_{b, v} & O_{b, w} \\
J_{c, k} & J_{c, g} & J_{c, h} & J_{c, u} & O_{c, v} & O_{c, w} \\
J_{d, k} & J_{d, g} & O_{d, h} & O_{d, u} & O_{d, v} & O_{d, w} \\
O_{e, k} & J_{e, g} & J_{e, h} & J_{e, u} & O_{e, v} & O_{e, w} \\
O_{f, k} & O_{f, g} & O_{f, h} & O_{f, u} & O_{f, v} & O_{f, w}
\end{array}\right],
$$


for some $a, b, c, d, e, f, g, h, k, u, v$ and $w$. Thus, with this notation,

$$
\bar{A}_{1}=\left[\begin{array}{cccccc}
J_{a, k} & J_{a, g} & J_{a, h} & O_{a, u} & J_{a, v} & O \\
J_{b, k} & J_{b, g} & J_{b, h} & O_{b, u} & J_{b, v} & O \\
O & O & O & O & O & O
\end{array}\right], \quad \bar{A}_{2}=\left[\begin{array}{ccc}
J_{a, k} & J_{a, g} & O \\
J_{b, k} & J_{b, g} & O \\
J_{c, k} & J_{c, g} & O \\
J_{d, k} & J_{d, g} & O \\
O & O & O
\end{array}\right]
$$

and

$$
\bar{A}_{3}=\left[\begin{array}{ccccc}
O_{a, k} & O_{a, g} & O_{a, h} & O_{a, u} & O_{a, v+w} \\
O_{b, k} & J_{b, g} & J_{b, h} & J_{b, u} & O_{b, v+w} \\
O_{c, k} & J_{c, g} & J_{c, h} & J_{c, u} & O_{c, v+w} \\
O_{d, k} & O_{d, g} & O_{d, h} & O_{d, u} & O_{d, v+w} \\
O_{e, k} & J_{e, g} & J_{e, h} & J_{e, u} & O_{e, v+w} \\
O_{f, k} & O_{f, g} & O_{f, h} & O_{f, u} & O_{f, v+w}
\end{array}\right] .
$$

Now, if

$$
\begin{aligned}
\bar{A}\left[r_{1}+1, \ldots, m \mid 1, \ldots, n\right]= & \bar{A}_{2}\left[r_{1}+1, \ldots, m \mid 1, \ldots, n\right] \\
& +\bar{A}_{3}\left[r_{1}+1, \ldots, m \mid 1, \ldots, n\right]
\end{aligned}
$$

has Boolean rank 1 then $d=e=0$ and hence $\bar{A}$ has the form

$$
\begin{aligned}
\bar{A} & =\left[\begin{array}{llllll}
J_{a, k} & J_{a, g} & J_{a, h} & O_{a, u} & J_{a, v} & O_{a, w} \\
J_{b, k} & J_{b, g} & J_{b, h} & J_{b, u} & J_{b, v} & O_{b, w} \\
J_{c, k} & J_{c, g} & J_{c, h} & J_{c, u} & O_{c, v} & O_{c, w} \\
O_{f, k} & O_{f, g} & O_{f, h} & O_{f, u} & O_{f, v} & O_{f, w}
\end{array}\right] \\
& =\left[\begin{array}{cccccc}
J_{a, k} & J_{a, g} & J_{a, h} & O_{a, u} & J_{a, v} & O \\
J_{b, k} & J_{b, g} & J_{b, h} & O_{b, u} & J_{b, v} & O \\
O & O & O & O & O & O
\end{array}\right]+\left[\begin{array}{cccccc}
O & O & O & O & O & O \\
J_{b, k} & J_{b, g} & J_{b, h} & J_{b, u} & O_{b, v} & O \\
J_{c, k} & J_{c, g} & J_{c, h} & J_{c, u} & O_{c, v} & O \\
O & O & O & O & O & O
\end{array}\right],
\end{aligned}
$$

so that $b(\bar{A})=2$, a contradiction to the assumption $b(\bar{A})=3$. Thus, $\bar{A}\left[r_{1}+1, \ldots\right.$, $m \mid 1, \ldots, n]$ must have Boolean rank 2 , and hence it has two isolated entries, say $i_{2}$ and $i_{3}$. If $\mathcal{C}_{1} \nsubseteq \mathcal{C}_{2} \cup \mathcal{C}_{3}$ then without loss of generality we have that $\bar{a}_{1, x} \neq 0$ for $x=k+g+h+u+1$, but then $\left\{\bar{a}_{1, x}, i_{2}, i_{3}\right\}$ is a set of three isolated entries, a contradiction to $\iota(\bar{A})=\iota(A)=2$. Thus, $v=0$ and hence, $\mathcal{C}_{1} \subseteq \mathcal{C}_{2} \cup \mathcal{C}_{3}$. Further, 
$\mathcal{C}_{1} \neq \mathcal{C}_{2} \cup \mathcal{C}_{3}$, otherwise $\bar{A}$ can be expressed as

$$
\bar{A}=\left[\begin{array}{ccc}
J_{a, k} & J_{a, g} & O \\
J_{b, k} & J_{b, g} & O \\
J_{c, k} & J_{c, g} & O \\
J_{d, k} & J_{d, g} & O \\
O & O & O
\end{array}\right]+\left[\begin{array}{cccc}
O_{a, k} & J_{a, g} & J_{a, h} & O \\
O_{b, k} & J_{b, g} & J_{b, h} & O \\
O_{c, k} & J_{c, g} & J_{c, h} & O \\
O_{d, k} & O_{d, g} & O_{d, h} & O \\
O_{e, k} & J_{e, g} & J_{e, h} & O \\
O_{f, k} & O_{f, g} & O_{f, h} & O
\end{array}\right]
$$

so that $b(\bar{A})=2$, contradiction to the assumption $b(\bar{A})=3$.

Note that $a, u, d \neq 0$, for otherwise $b(\bar{A})=2$. If $e=0$ then $b+c \neq 0$ so that $\left\{\bar{a}_{1, c_{1}}, \bar{a}_{a+1, k+c_{3}}, \bar{a}_{r_{2}, 1}\right\}$ is a set of three isolated entries, a contradiction to $\iota(\bar{A})=$ $\iota(A)=2$. If $e \neq 0$, then $\left\{\bar{a}_{1, c_{1}}, \bar{a}_{r_{2}, 1}, \bar{a}_{r_{2}+e, k+c_{3}}\right\}$ is a set of three isolated entries, contradicting that $\iota(\bar{A})=\iota(A)=2$. Thus, $\mathcal{R}_{1} \not \subset \mathcal{R}_{2}$.

By renumbering or transposing we have proved that $\mathcal{R}_{i} \not \subset \mathcal{R}_{j}$ and $\mathcal{C}_{i} \not \subset \mathcal{C}_{j}$ for any pair $i$ and $j$.

Now, permute the rows and columns of $\bar{A}$ so that

$$
\begin{aligned}
\mathcal{R}_{1}= & \left\{1,2, \ldots, r_{1}\right\}, \\
\mathcal{R}_{2}= & \{a+1, a+2, \ldots, a+b, a+b+c+1, a+b+c+2, \ldots, \\
& a+b+c+d+e+f\},
\end{aligned}
$$

and

$$
\begin{gathered}
\mathcal{R}_{3}=\{a+b+1, a+b+2, \ldots, a+b+c+d+e, a+b+c+e+f+1, \\
a+b+c+e+f+2, \ldots, a+b+c+e+f+g\}
\end{gathered}
$$

for some $a, b, c, d, e, f, g$ where $a+b+c+d=r_{1}$, so that $\bar{A}$ has the form

$$
\bar{A}=\left[\begin{array}{cccccccc}
J_{a, k} & O_{a, l} & J_{a, p} & O_{a, q} & J_{a, r} & O_{a, s} & J_{a, v} & O_{a, w} \\
J_{b, k} & J_{b, l} & J_{b, p} & J_{b, q} & J_{b, r} & O_{b, s} & J_{b, v} & O_{b, w} \\
J_{c, k} & O_{c, l} & J_{c, p} & J_{c, q} & J_{c, r} & J_{c, s} & J_{c, v} & O_{c, w} \\
J_{d, k} & J_{d, l} & J_{d, p} & J_{d, q} & J_{d, r} & J_{d, s} & J_{d, v} & O_{d, w} \\
J_{e, k} & J_{e, l} & J_{e, p} & J_{e, q} & J_{e, r} & J_{e, s} & O_{e, v} & O_{e, w} \\
J_{f, k} & J_{f, l} & J_{f, p} & J_{f, q} & O_{f, r} & O_{f, s} & O_{f, v} & O_{f, w} \\
O_{g, k} & O_{g, l} & J_{g, p} & J_{g, q} & J_{g, r} & J_{g, s} & O_{g, v} & O_{g, w} \\
O_{h, k} & O_{h, l} & O_{h, p} & O_{h, q} & O_{h, r} & O_{h, s} & O_{h, v} & O_{h, w}
\end{array}\right],
$$


for some $a, b, c, d, e, f, g, h, k, l, p, q, r, s, v$, and $w$, so that

$$
\begin{aligned}
\bar{A}_{1}= & {\left[\begin{array}{cccccccc}
J_{a, k} & O_{a, l} & J_{a, p} & O_{a, q} & J_{a, r} & O_{a, s} & J_{a, v} & O_{a, w} \\
J_{b, k} & O_{b, l} & J_{b, p} & O_{b, q} & J_{b, r} & O_{b, s} & J_{b, v} & O_{b, w} \\
J_{c, k} & O_{c, l} & J_{c, p} & O_{c, q} & J_{c, r} & O_{c, s} & J_{c, v} & O_{c, w} \\
J_{d, k} & O_{d, l} & J_{d, p} & O_{d, q} & J_{d, r} & O_{d, s} & J_{d, v} & O_{d, w} \\
O & O & O & O & O & O & O & O
\end{array}\right], } \\
\bar{A}_{2}= & {\left[\begin{array}{ccccc}
O_{a, k} & O_{a, l} & O_{a, p} & O_{a, q} & O \\
J_{b, k} & J_{b, l} & J_{b, p} & J_{b, q} & O \\
O_{c, k} & O_{c, l} & O_{c, p} & O_{c, q} & O \\
J_{d, k} & J_{d, l} & J_{d, p} & J_{d, q} & O \\
J_{e, k} & J_{e, l} & J_{e, p} & J_{e, q} & O \\
J_{f, k} & J_{f, l} & J_{f, p} & J_{f, q} & O \\
O & O & O & O & O
\end{array}\right], }
\end{aligned}
$$

and

$$
\bar{A}_{3}=\left[\begin{array}{ccccccc}
O_{a, k} & O_{a, l} & O_{a, p} & O_{a, q} & O_{a, r} & O_{a, s} & O \\
O_{b, k} & O_{b, l} & O_{b, p} & O_{b, q} & O_{b, r} & O_{b, s} & O \\
O_{c, k} & O_{c, l} & J_{c, p} & J_{c, q} & J_{c, r} & J_{c, s} & O \\
O_{d, k} & O_{d, l} & J_{d, p} & J_{d, q} & J_{d, r} & J_{d, s} & O \\
O_{e, k} & O_{e, l} & J_{e, p} & J_{e, q} & J_{e, r} & J_{e, s} & O \\
O_{f, k} & O_{f, l} & O_{f, p} & O_{f, q} & O_{f, r} & O_{f, s} & O \\
O_{g, k} & O_{g, l} & J_{g, p} & J_{g, q} & J_{g, r} & J_{g, s} & O \\
O_{h, k} & O_{h, l} & O_{h, p} & O_{h, q} & O_{h, r} & O_{h, s} & O
\end{array}\right] .
$$

Suppose that $v \neq 0$ and $\bar{A}\left[r_{1}+1, \ldots, m \mid 1, \ldots, n\right]=\bar{A}_{2}\left[r_{1}+1, \ldots, m \mid 1, \ldots, n\right]+$ $\bar{A}_{3}\left[r_{1}+1, \ldots, m \mid 1, \ldots, n\right]$ has Boolean rank 1. Then $f=g=0$ and we must have $l, s \neq 0$, for otherwise $b(\bar{A})=2$, a contradiction. Further, if $b=c=0$ then $b(\bar{A})=2$, again a contradiction. Thus, using a 1 from each of the blocks subscripted $(a, v)$, $(b, l)$ and $(e, s)$ of $\bar{A}$ or a 1 from each of the blocks subscripted $(a, v),(e, l)$ and $(c, s)$ of $\bar{A}$ we have three isolated entries, a contradiction since $\iota(A)=\iota(\bar{A})=2$. Thus the Boolean rank of $\bar{A}\left[r_{1}+1, \ldots, m \mid 1, \ldots, n\right]$ must be 2 , and hence it has two isolated entries, say $i_{2}$ and $i_{3}$. If $\mathcal{C}_{1} \nsubseteq \mathcal{C}_{2} \cup \mathcal{C}_{3}$ then $\bar{a}_{1, x} \neq 0$ for $x=k+l+p+q+r+s+1$ and then $\left\{\bar{a}_{1, x}, i_{2}, i_{3}\right\}$ is a set of three isolated entries, a contradiction to $\iota(A)=\iota(\bar{A})=2$. Thus, $\mathcal{C}_{1} \subseteq \mathcal{C}_{2} \cup \mathcal{C}_{3}$. Further, $\mathcal{C}_{1} \neq \mathcal{C}_{2} \cup \mathcal{C}_{3}$, otherwise, $\bar{A}$ would have Boolean rank 2 . Thus, $v=0$, and hence, $\mathcal{C}_{1} \subset \mathcal{C}_{2} \cup \mathcal{C}_{3}$.

Since the choice of rows versus columns can be changed by transposition and the index of $\mathcal{R}_{i}$ and $\mathcal{C}_{j}$ by renumbering, we have shown that if $\{i, j, k\}=\{1,2,3\}$ then $\mathcal{R}_{i} \subset \mathcal{R}_{j} \cup \mathcal{R}_{k}$ and $\mathcal{C}_{i} \subset \mathcal{C}_{j} \cup \mathcal{C}_{k}$. 
Consider the matrix (1). Since $\mathcal{R}_{1} \subset \mathcal{R}_{2} \cup \mathcal{R}_{3}$ we have that $a=0$; since $\mathcal{R}_{2} \subset$ $\mathcal{R}_{1} \cup \mathcal{R}_{3}$ we have that $f=0$; since $\mathcal{C}_{2} \subset \mathcal{C}_{1} \cup \mathcal{C}_{3}$ we have that $l=0$; and since $\mathcal{C}_{3} \subset \mathcal{C}_{1} \cup \mathcal{C}_{2}$ we have that $s=0$. That is, since $a=f=l=s=v=0, \bar{A}$ has the form

$$
\bar{A}=\left[\begin{array}{lllll}
J & J & J & J & O \\
J & J & J & J & O \\
J & J & J & J & O \\
J & J & J & J & O \\
O & J & J & J & O \\
O & O & O & O & O
\end{array}\right],
$$

where the indices have been omitted. Thus $b(\bar{A})=2$, a contradiction. Thus, if $\iota(A)=2$ then $b(\bar{A}) \neq 3$.

Theorem 2.8. Let $A \in \mathcal{M}_{m, n}\left(\mathbb{Z}_{+}\right)$. Then $\iota(A)=2$ if and only if $b(\bar{A})=2$.

P r o of. From Lemma 2.7, we have the sufficiency. So we only need to show the necessity.

Suppose there exists $A \in \mathcal{M}_{m, n}\left(\mathbb{Z}_{+}\right)$with $\iota(A)=\iota(\bar{A})=2$ and $b(\bar{A})>2$. By Lemma 2.7, $b(\bar{A}) \neq 3$, and hence $b(\bar{A}) \geqslant 4$. Thus we choose $A$ such that if $b(\bar{A})>$ $r_{\mathbb{B}}(\bar{C})>2$ then $\iota(C)>2$. Suppose that $\bar{A}=\bar{A}_{1}+\bar{A}_{2}+\ldots+\bar{A}_{k}$ for $k=b(\bar{A})$ where each $\bar{A}_{i}$ is Boolean rank one, i.e., $k$ is the minimum $k$ such that $b(\bar{A})=k$ and $\iota(A)=2$. Suppose that $\bar{A}_{1}$ has the fewest number of nonzero rows of the $\bar{A}_{i}$ 's. As in the proof of the above Lemma 2.7, permute the rows of $\bar{A}$ so that $\bar{A}_{1}$ has nonzero rows $1,2, \ldots, r_{1}$. For $j=1, \ldots, r_{1}$, let $\bar{B}_{j}$ be the matrix whose first $j$ rows are the first $j$ rows of $\bar{A}$ and whose last $m-j$ rows are all zero. Let $\bar{C}_{j}$ be the matrix whose first $j$ rows are all zero and whose last $m-j$ rows are the last $m-j$ rows of $\bar{A}$. Then $\bar{A}=\bar{B}_{j}+\bar{C}_{j}$. Further, any set of isolated entries of $\bar{C}_{j}$ is a set of isolated entries for $\bar{A}$. Now, $b(\bar{A}) \leqslant b\left(\bar{B}_{j}\right)+b\left(\bar{C}_{j}\right)$, and the fact that $b\left(\bar{C}_{j}\right)=b\left(\bar{C}_{j-1}\right)$ or $b\left(\bar{C}_{j}\right)=b\left(\bar{C}_{j-1}\right)-1$ imply that there is some $t$ such that $b\left(\bar{C}_{t}\right)=b(\bar{A})-1$. Since $b\left(\bar{C}_{t}\right)<k$ by the choice of $\bar{A}$, for this $t$, we have that $\iota\left(\bar{C}_{t}\right)>2$ since $b\left(\bar{C}_{t}\right) \geqslant 3$. That is, $\iota(A)=\iota(\bar{A})>2$, a contradiction.

Now, as we can see in the following example, there is a matrix $A \in \mathcal{M}_{m, n}\left(\mathbb{Z}_{+}\right)$ such that $\iota(\bar{A})=3$ and $b(\bar{A})$ is relative large, depending on $m$ and $n$.

Example 2.9. For $n \geqslant 3$, let $\bar{D}_{n}=J \backslash I \in \mathcal{M}_{n}(\mathbb{B})$. Then it is easily shown that $\iota\left(\bar{D}_{n}\right)=3$ while $b\left(\bar{D}_{n}\right)=k$ where $k=\min \left\{k: n \leqslant\left(\begin{array}{c}k \\ k / 2\end{array}\right)\right\}$, see [5], Corollary 2. So, $\iota\left(\bar{D}_{20}\right)=3$ while $b\left(\bar{D}_{20}\right)=6$.

A tournament matrix $[T] \in \mathcal{M}_{n}(\mathbb{B})$ is the adjacency matrix of a directed graph called a tournament, $T$. It is characterized by $[T] \circ[T]^{t}=O$ and $[T]+[T]^{t}=J-I$. 
Now, it is natural to ask the following: How much difference can there be between the isolation number of a matrix in $\mathcal{M}_{m, n}\left(\mathbb{Z}_{+}\right)$and the Boolean rank of its support matrix? For each $k=1,2, \ldots, \min \{m, n\}$ characterizes the matrices in $\mathcal{M}_{m, n}\left(\mathbb{Z}_{+}\right)$ for which $\iota(A)=b(\bar{A})$. Of course this is done if $k=1$ or $k=2$ in the above theorems, but only in those cases. For $k=m$ we can also find a characterization:

Theorem 2.10. Let $1 \leqslant m \leqslant n$ and $A \in \mathcal{M}_{m, n}\left(\mathbb{Z}_{+}\right)$. Then $\iota(A)=b(\bar{A})=m$ if and only if there exist permutation matrices $P \in \mathcal{M}_{m}(\mathbb{B})$ and $Q \in \mathcal{M}_{n}(\mathbb{B})$ such that $P A Q=[B \mid C]$ where $\bar{B}=I_{m}+\bar{T} \in \mathcal{M}_{m}(\mathbb{B})$ and $\bar{T} \in \mathcal{M}_{m}(\mathbb{B})$ is dominated by a tournament matrix. (There are no restrictions on $C$.)

P r o of. Suppose that $\iota(A)=m$. Then we permute $A$ by permutation matrices $P$ and $Q$ so that the isolated entries are in the $(d, d)$ positions, $d=1, \ldots, m$. That is, if $X=P A Q$ then $I=\left\{x_{1,1}, x_{2,2}, \ldots, x_{m, m}\right\}$ is the set of isolated entries in $\mathrm{X}$. Therefore $X=[B \mid C]$, with $\bar{b}_{i, i}=\bar{x}_{i, i}=1$ and $\bar{b}_{i, j} \cdot \bar{b}_{j, i}=0$ for every $i$ and $j \neq i$ from the definition of the isolated entries. Thus, $\bar{B}=I_{m}+\bar{T}$ where $\bar{T}$ is an $m$ square matrix which is dominated by a tournament matrix. Thus, $P A Q=[B \mid C]$ where $\bar{B}=I_{m}+\bar{T}$ and clearly there are no conditions on $C$.

Conversely, if $P A Q=[B \mid C]$ and $\bar{B}=I_{m}+\bar{T}$ where $\bar{T}$ is an $m$ square matrix which is dominated by a tournament matrix, then the diagonal entries of $B$ form a set of isolated entries for $P A Q$ and hence $A$ has a set of $m$ isolated entries. Thus $\iota(A)=b(\bar{A})=m$.

Corollary 2.11. Let $1 \leqslant m \leqslant n$ and $A \in \mathcal{M}_{m, n}\left(\mathbb{Z}_{+}\right)$. If there exist permutation matrices $P \in \mathcal{M}_{m}(\mathbb{B})$ and $Q \in \mathcal{M}_{n}(\mathbb{B})$ such that $P A Q=[B \mid C]$ where $B \in \mathcal{M}_{m}\left(\mathbb{Z}_{+}\right)$ is a diagonal matrix or a triangular matrix with nonzero diagonal entries, then $\iota(A)=$ $b(\bar{A})=m$.

\section{Conclusion}

We considered the isolation number of a matrix $A$ over $\mathbb{Z}_{+}$and compared it with the Boolean rank of the support $\bar{A}$ of $A$. We determined that the isolation number and the Boolean rank of the support of a matrix $A$ are the same if and only if the isolation number is 1 or 2 only. We also determined a special type of $m \times n$ matrices whose isolation number is $m$. That is, those matrices are permutationally equivalent to a matrix $A$ whose support contains a submatrix of a sum of the identity matrix and a tournament matrix.

In further research on isolation numbers of matrices over $\mathbb{Z}_{+}$we will apply these results to characterize the linear operators that preserve the isolation numbers of matrices in $\mathcal{M}_{m, n}\left(\mathbb{Z}_{+}\right)$. 
Acknowledgement. The authors are highly grateful to the referee for valuable comments and suggestions helpful in improving this paper.

\section{References}

[1] L. B. Beasley: Isolation number versus Boolean rank. Linear Algebra Appl. 436 (2012), 3469-3474.

[2] L.B.Beasley, D.A. Gregory, N. J.Pullman: Nonnegative rank-preserving operators. Linear Algebra Appl. 65 (1985), 207-223.

[3] J.A.Bondy, U.S.R.Murty: Graph Theory. Graduate texts in Mathematics 244, Springer, Berlin, 2008.

[4] R. A. Brualdi, H. J. Ryser: Combinatorial Matrix Theory. Encyclopedia of Mathematics and Its Applications 39, Cambridge University Press, Cambridge, 1991.

[5] D. de Caen, D. A. Gregory, N. J. Pullman: The Boolean rank of zero-one matrices. Combinatorics and Computing. Proc. 3rd Caribb. Conf., Cave Hill/Barbados, 1981, pp. $169-173$.

[6] D. A. Gregory, N. J. Pullman, K. F. Jones, J. R. Lundgren: Biclique coverings of regular bigraphs and minimum semiring ranks of regular matrices. J. Comb. Theory, Ser. B 51 (1991), 73-89.

zbl MR doi

7] A. Kato: Complexity of the sex-equal stable marriage problem. Japan J. Ind. Appl. Math. 10 (1993), 1-19.

[8] G. Markowsky: Primes, irreducibles and extremal lattices. Order 9 (1992), 265-290.

$\mathrm{zbl} \mathrm{MR}$

zbl MR doi

zbl MR doi

zbl MR doi

Authors' addresses: Le R oy B. Beasley, Department of Mathematics and Statistics, Utah State University, Logan, Utah 84322-3900, USA, e-mail: leroy.b.beasley๔ aggiemail.usu.edu; Young B a e J un, Department of Mathematics Education, Gyeongsang National University, Jinju 52828, Korea, e-mail: ybjun@gsnu.ac.kr; Seok-Zun S o n g (corresponding author), Department of Mathematics, Jeju National University, Jeju 63243, Korea, e-mail: szsong@jejunu.ac.kr. 